\title{
Cicatrización por segunda intención en carcinomas basocelulares extensos luego de la cirugía micrográfica de Mohs: a propósito de un caso clínico
}

\author{
Evelyn Ruiz ${ }^{*}$, Julio Magliano ${ }^{\dagger}$ Carlos Bazzano ${ }^{\ddagger}$
}

\section{Resumen}

La cirugía micrográfica de Mohs es una técnica que se utiliza para la exéresis de distintos tipos de cánceres de piel que permite el examen histológico del $100 \%$ de los márgenes quirúrgicos, con máxima preservación de tejido sano y logrando las más altas tasas de curación. Luego de la realización de la misma existen distintas técnicas para reparar el defecto resultante, una de estas es la cicatrización por segunda intención; consiste en dejar granular la herida de forma espontánea, se puede asistir a esta técnica con apósitos de hidrocoloides para favorecer la cicatrización.

Se describe un caso de una paciente añosa con tres defectos grandes faciales, donde se optó por dejar cicatrizar por segunda intención asistido con apósitos hidrocoloidales.

Palabras clave: Cirugía de Mohs Carcinoma basocelular Cicatrización por segunda intención

Key words: $\quad$ Mohs Surgery Carcinoma, Basal Cell Healing by second intention

\section{Introducción}

La cirugía micrográfica de Mohs (CMM) es una técnica quirúrgica en etapas con control histológico del 100\% de los márgenes; entre sus indicaciones encontramos el carcinoma basocelular (CBC) de alto riesgo, así como otros tumores de piel ${ }^{(1)}$.

En las primeras 130 intervenciones realizadas en nuestro servicio, $68 \%$ correspondieron a $\mathrm{CBC}$ y dentro de estos, los subtipos histopatológicos fueron: nodular $89,8 \%$, superficial $3,4 \%$, infiltrativo $3,4 \%$, adenoide $2,3 \%$ y micronodular $1,1 \%^{(2)}$.

Cuando hablamos de riesgo de recidiva nos referimos al riesgo de compromiso de estructuras nobles que no estaban comprometidas antes de la recurrencia. Para valorar el riesgo se combinan distintos factores, como son la asociación de topografía y tamaño del tumor así como las formas clínicas, características histológicas y terreno del paciente.

Con respecto a la topografía definimos zona de alto riesgo o área $\mathrm{H}$ la comprendida en las regiones de máscara facial (cara central, párpados, canto interno y externo de ojos, cejas, zona periorbitaria, nariz, labios, mentón, mandíbula, región preauricular y posauricular, orejas) genitales, zonas perineal, pezones, areola, manos y pies. La zona de mediano riesgo o área $\mathrm{M}$ es la comprendida por mejillas, frente, cuero cabelludo, cuello y zona pretibial y la de bajo riesgo o área $\mathrm{L}$, la región de tronco y extremidades excluyendo manos, pies y tobillos ${ }^{(3)}$.

* Residente de Dermatología.

† Médico dermatólogo. Prof. Adjunto de Cátedra. Jefe de la Unidad de Cirugía Dermatológica de la Cátedra de Dermatología.

‡ Médico dermatólogo. Prof. Agregado de Cátedra, coordinador general de Cirugía Dermatológica.

Unidad de Cirugía Dermatológica. Cátedra de Dermatología Médico Quirúrgica. Hospital de Clínicas. Universidad de la República. Montevideo,

Uruguay.

Los autores declaran no tener conflicto de intereses.

Recibido: 19/3/18

Aprobado: $11 / 6 / 18$ 

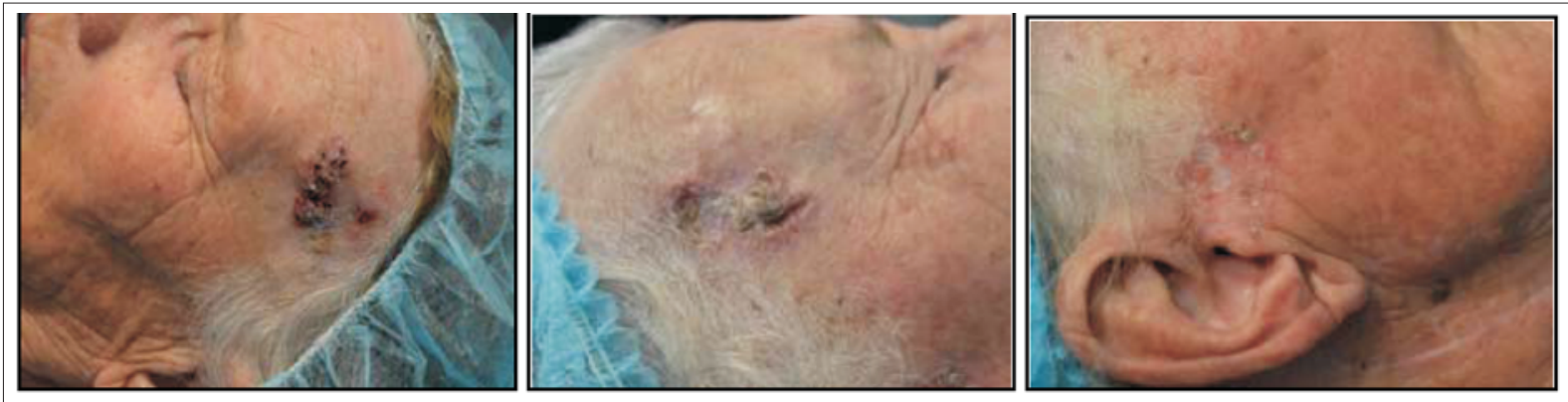

Figura 1. Carcinomas basocelulares faciales antes de la cirugía micrográfica de Mohs.

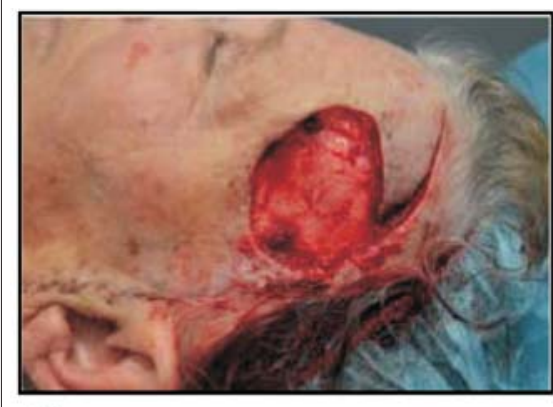

A)

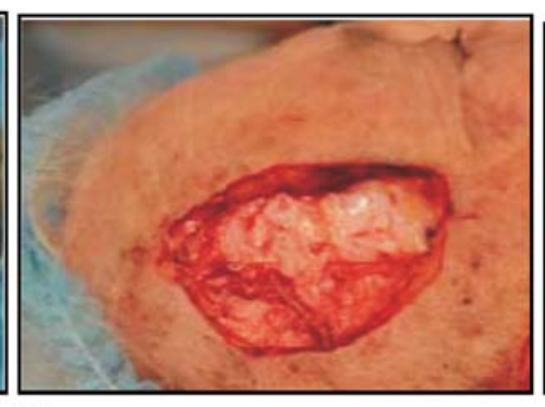

B)

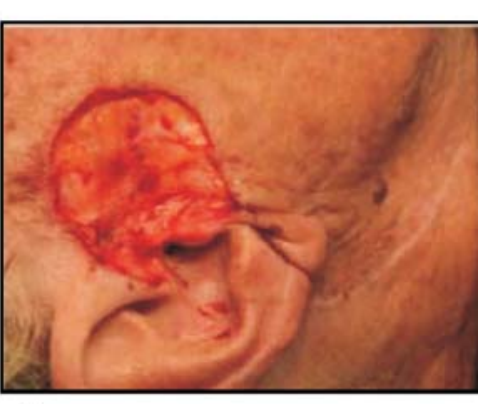

C)

Figura 2. Defecto final luego de cirugía micrográfica de Mohs (CMM). A) Defecto de 5,8 por 6,5 cm en región temporal izquierda. B) Defecto final de 6,4 por 4,4 cm en región temporal derecha. C) Defecto de 4,0 por 4,2 cm localizado en región preauricular derecha luego de CMM.

Definimos entonces un tumor como de alto riesgo cuando está localizado en zonas L y que mide $20 \mathrm{~mm}$ o más, los localizados en zona $\mathrm{M}$ mayores o iguales a 10 mm y los que se encuentran en zona $\mathrm{H}$, sin importar en esta última el tamaño del mismo, según una actualización reciente de las guías de la National Comprehensive Cancer Network $(\mathrm{NCCN})^{(4)}$.

Los límites mal definidos, al igual que las formas histológicas infiltrativas, metatípicas, esclerosantes con infiltración mixta o micronodular en cualquier parte del tumor, la invasión perineural, los tumores recidivantes, los que se encuentran en piel irradiada previamente, en pacientes inmunodeprimidos o los que recibieron tratamiento primario incompleto se consideran de alto ries$\mathrm{go}^{(5)}$.

Luego de realizarse la CMM el defecto resultante puede reconstituirse mediante el cierre simple borde a borde, por el uso de colgajos locales, injertos o el cierre por segunda intención.

En la cicatrización por segunda intención los defectos cutáneos se dejan granular de forma espontánea, la misma es una forma simple y rentable de cierre de la herida que implica cuidados ambulatorios posoperatorios básicos, presenta baja tasa de infección y preserva la arquitectura local de la piel ${ }^{(6)}$.

La elección dependerá del tamaño del defecto y de las condiciones propias del paciente.

\section{Caso clínico}

Mujer de 86 años de edad, fototipo II de la clasificación de Fitzpatrick, con antecedentes personales de múltiples carcinomas basocelulares de siete años de evolución resueltos quirúrgicamente.

Consulta en servicio de dermatología por tres lesiones tumorales en cara localizadas en región temporal izquierda, región preauricular derecha y temporal derecha, de 3,5 por $4,5 \mathrm{~cm} ; 3,2$ por $2,2 \mathrm{~cm}, \mathrm{y} 3,4$ por $4,5 \mathrm{~cm}$, respectivamente, eritematosas con costras hemáticas en su superficie e infiltradas a la palpación, dolorosas, de años de evolución (figura 1).

Con el planteo clínico de $\mathrm{CBC}$ se realizó biopsia de las tres lesiones confirmando el diagnóstico.

El tratamiento de elección fue la CMM en las tres lesiones y los defectos se dejaron cicatrizar por segunda intención (figuras 2 y 3). La cicatrización fue asistida con apósitos de hidrocoloides, los cuales se cambiaron semanalmente. A los 20 días del posoperatorio se sus- 


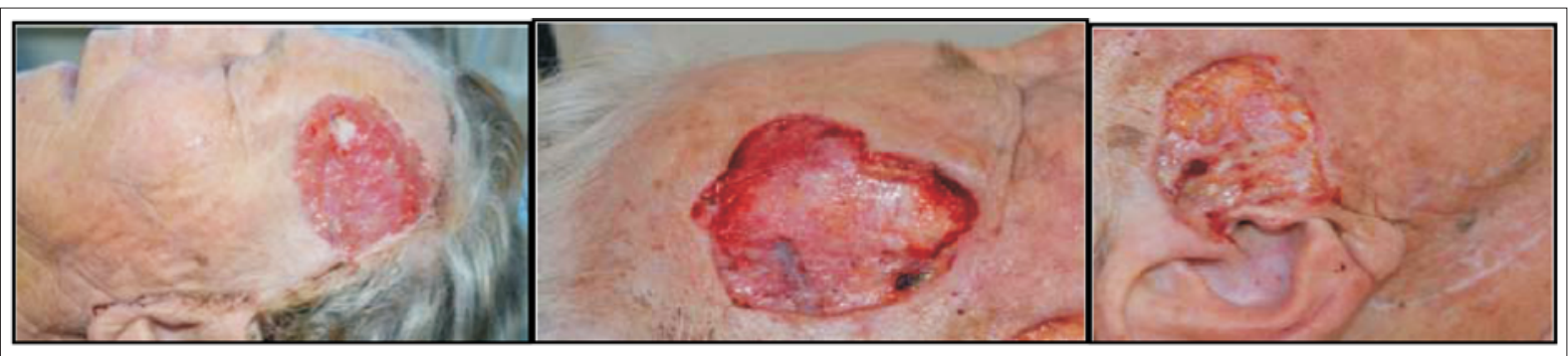

Figura 3. Cierre por segunda intención, a un mes del posoperatorio.

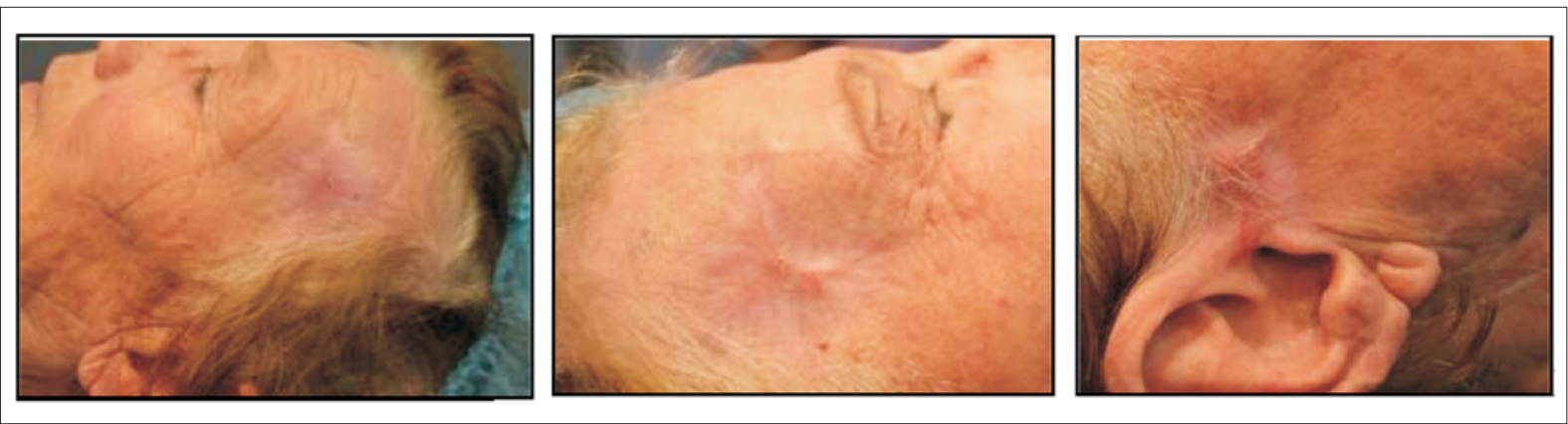

Figura 4. Muestra la cicatrización por segunda intención luego de seis meses de la cirugía micrográfica de Mohs.

pendieron los apósitos y se indicaron curaciones con sulfadiacina de plata en crema.

Se realizó control a los seis meses luego de la cirugía con excelentes resultados estéticos y funcionales (figura 4).

\section{Discusión}

El cáncer de piel constituye el tumor maligno más frecuente en caucásicos, siendo un tercio del total de las neoplasias. Es considerable el aumento de su incidencia en todos los países, excepto en Australia. El carcinoma basocelular es el más frecuente y representa el $75 \%$ de los cánceres de piel no melanoma (CPNM), afecta al $30 \%$ de la población mundial y su incidencia aumenta año a año ${ }^{(7)}$.

En nuestro país, según la Comisión Honoraria de Lucha contra el Cáncer, entre los años 2007 y 2011 el promedio anual de CPNM fue de 2.000 casos, la incidencia no es del todo ajustada debido a que en la mayoría de los casos no se realiza un registro adecuado ${ }^{(8)}$. En cuanto a la mortalidad del CPNM en el período de 2009-2013 para los hombres fue de 0,95 casos por 100.000 habitantes, correspondiendo 0,62 casos por 100.000 a carcinoma espinocelular y 0,16 casos por $100.000 \mathrm{a} \mathrm{CBC}^{(9)}$.

La localización más común es cabeza y cuello (83\%), le siguen tronco y extremidades (26\%). La piel clara y la exposición solar intermitente juegan un papel fundamental en el desarrollo de $\mathrm{CBC}^{(10)}$. Magliano y colaboradores reportan con respecto a la distribución de los $\mathrm{CBC}$ operados por CMM, específicamente en la cabeza, que $24,4 \%$ se localizaron en nariz, $21,4 \%$ en región periorbitaria, $13 \%$ en mejilla, entre otras ${ }^{(2)}$. El CBC es un carcinoma que crece lentamente y los casos de metástasis son poco frecuentes, ocurren en solo $0,003 \%$ a $0,05 \%$ de los pacientes, y pueden ocurrir más comúnmente a través de los vasos linfáticos a los ganglios regionales ${ }^{(11)}$. Puede comprometer órganos nobles, sobre todo los de localización facial.

La elección del tratamiento depende de varios factores, como son el tipo tumoral y la localización anatómica del mismo, de la edad y del estado general del paciente así como de los recursos sanitarios y la experiencia del dermatólogo.

El objetivo principal del tratamiento es la remoción completa del tumor en el primer acto terapéutico; como objetivos secundarios, subordinados a nuestro objetivo principal, consideramos conservar la función, la forma y los aspectos estéticos ${ }^{(12)}$.

Existen varias opciones terapéuticas para los CPNM, donde se incluyen opciones que no tienen control de los márgenes; la radioterapia, la criocirugía, el curetaje y la electrodesecación, la quimioterapia tópica, el imiquimod, el interferón y la terapia fotodinámica. En cambio, las opciones terapéuticas con control de márgenes son la cirugía convencional y la CMM. Para el grupo de pacientes que no son candidatos a las terapias convencionales, actualmente contamos con fármacos como vismodegib y erismodegib/sonidegib, aprobados por la 
Food and Drug Administration, ambos inhibidores de la vía Hedgehog, vía implicada en el desarrollo de diversas neoplasias, entre ellas el CBC. Están indicados para pacientes con CBC metastásico, $\mathrm{CBC}$ localmente avanzado, recurrente luego de cirugía, o que no es candidato para cirugía o radioterapia ${ }^{(13,14)}$.

En el caso de nuestra paciente, que presenta tres tumores de alto riesgo con grandes áreas faciales comprometidas y con dolor local, la técnica deber ser quirúrgica con control de márgenes y de estar disponible lo ideal es la CMM. Esta modalidad quirúrgica se fundamenta en el hecho de que el cáncer de piel tiene un crecimiento continuo con extensión microscópica más allá de los márgenes clínicamente identificables. A través de la escisión del tumor capa por capa y la observación microscópica de los márgenes y del lecho quirúrgico, es posible extirpar de forma completa el tumor $^{(3)}$.

La cirugía de Mohs es un tratamiento pretendidamente curativo, es una técnica quirúrgica que permite el control histológico del $100 \%$ de los márgenes, formalmente indicado para la resección de tumores de alto riesgo, que permite el análisis del $100 \%$ de los márgenes del tumor y conservar al máximo el tejido sano, lo que hace de ella una técnica que obtiene los más elevados porcentajes de curación ${ }^{(10)}$.

Luego de finalizada la exéresis quirúrgica del tumor se debe plantear la reparación del defecto quirúrgico con la finalidad de obtener los mejores resultados funcionales, anatómicos y estéticos.

Existen varias técnicas para la reconstrucción local: cierre directo borde a borde, colgajos locales injertos o cierre por segunda intención. La mayoría de los defectos cutáneos son aptos para ser corregidos con algunas de estas técnicas.

Nuestra experiencia con el cierre de los defectos en las primeras $130 \mathrm{CMM}$ : cerrados a través de colgajos locales en $43 \%$; cierre simple en $35 \%$, y en $16,5 \%$ se empleó la cicatrización por segunda intención ${ }^{(2)}$.

El cierre simple o cierre directo es el cierre borde a borde del defecto, esta técnica redistribuye la tensión, evierte el borde de la herida, elimina el espacio muerto y mantiene o restablece los contornos anatómicos naturales, a la vez que minimiza la formación de marcas permanentes de la sutura en la piel ${ }^{(15)}$.

Dado el tamaño de los defectos en nuestra paciente, no es una opción válida de realizar.

Un colgajo es una porción de piel de espesor completo que se transfiere de un sitio donante para cubrir el defecto, manteniendo un pedículo vascular que lo une de su lecho primitivo ${ }^{(16)}$.
Por el tamaño y la localización de los defectos quirúrgicos no la consideramos como la mejor opción quirúrgica.

Los injertos cutáneos son fragmentos de piel de espesor variable que se obtienen de una zona de piel (zona donante) con características similares a la zona a tratar (zona receptora) con el fin de cubrir un defecto. Dentro de sus indicaciones están: el recubrimiento de los defectos de forma permanente o temporal, reducir la contracción de una cicatriz previa, acelerar la cicatrización de heridas o quemaduras ${ }^{(17)}$.

Consisten en una buena opción en nuestra paciente pero implican crear nuevos defectos quirúrgicos y requieren cuidados adecuados de inmovilización frente al riesgo de pérdida parcial o total de los mismos.

En el caso de esta paciente, debido a su edad, el gran tamaño de los defectos finales y la localización de los mismos, optamos por la cicatrización por segunda intención asistida por apósitos de hidrocoloides. La misma consiste en permitir que la herida granule y luego cicatrice por sí sola.

No se necesita ninguna intervención más que los cuidados locales y controlar que el proceso de cicatrización de la herida sea el adecuado, manteniendo la herida limpia, húmeda y minimizando la carga bacteriana ${ }^{(18)}$.

El resultado estético con la cicatrización por segunda intención es inferior al obtenido por el cierre simple o con el uso de colgajos locales, como se reporta en un estudio realizado en nuestro servicio ${ }^{(19)}$.

El proceso puede realizarse simplemente a través de la evolución natural de cicatrización o asistida con algún tipo de apósito, entre los que están los apósitos hidrocoloidales.

Los apósitos de hidrocoloides están formados por carboximetilcelulosa sódica, pectina, gelatina y adhesivos que asientan sobre un film semipermeable de espuma. Esto genera un apósito oclusivo y adherente que forma un gel sobre la superficie a tratar, son permeables al oxígeno y a la humedad pero no a bacterias y fluidos, manteniendo la cicatrización en un ambiente húmedo ${ }^{(20)}$.

El mecanismo de acción de los apósitos hidrocoloidales se basa en que si la superficie de la herida se mantiene húmeda, por ejemplo cubriéndola con una película oclusiva, se suprime la formación de escaras, hay menos pérdida de células y fluidos, hay un menor grado de cambios inflamatorios, aumentando la tasa de reepitelialización ${ }^{(21)}$.

El uso de apósitos de forma correcta ofrece ventajas sobre el posoperatorio convencional al disminuir el dolor en algunos casos, reducir la incomodidad, el edema, enrojecimiento y secreción, así como a largo plazo generando cicatrices más pequeñas, que generan menos de- 
formidades anatómicas, dando una mejor apariencia cosmética.

La principal desventaja de los mismos es el cambio frecuente que requieren, principalmente al principio del tratamiento, lo que puede generar incomodidad para el paciente ${ }^{(20,21)}$.

En el caso de nuestra paciente la cicatrización por segunda intención, en ambiente húmedo, proporcionado por los apósitos hidrocoloidales, posibilitó una cicatriz de buena calidad, con escasa retracción y de una coloración muy similar a la de la piel circundante, produciendo un efecto estético bueno.

\section{Conclusión}

En determinadas circunstancias en la que luego de la CMM quedan defectos quirúrgicos de gran tamaño o que comprometen grandes sitios anatómicos y que por alguna determinada razón la reconstrucción por medio de cierre simple o colgajos no es posible, o es dificultosa, puede optarse por injertos o cicatrización por segunda intención.

En algunos de estas situaciones, como en el caso de nuestra paciente, con la cicatrización por segunda intención se obtienen resultados superiores a los injertos con muchas menos complicaciones y mejores resultados estéticos.

Cuando esta técnica es asistida por apósitos de hidrocoloides facilita la cicatrización de buena calidad, con escasa retracción y color similar a la piel circundante, logrando muy buenos resultados estéticos, minimizando las molestias, además de que permite disminuir el número de controles logrando una buena relación costo-beneficio.

\section{Abstract}

Mohs surgery is a technique used for the excision of different types of skin cancer which enabled the histological study of the entire $(100 \%)$ surgical margins, with maximum preservation of healthy tissue and leading to the highest healing rates. After surgery, several techniques are available to repair resulting defects, one of which is healing by second intention. The latter consists in allowing granulation tissue to be formed spontaneously, which can be assisted with hydrocolloid dressings to favor healing.

The case of an old age female patient with three facial defects is described. Second intention healing was the choice, assisting it with hydrocolloid dressings.

\section{Resumo}

A cirurgia micrográfica de Mohs é uma técnica utilizada na exérese de distintos tipos de cânceres de pele, que permite o exame histológico de $100 \%$ das margens ci- rúrgicas, com máxima preservação do tecido saudável conseguindo as taxas mais altas de cura. Depois da realização da mesma existem distintas técnicas para reparar o defeito resultante, sendo uma delas a cicatrização por segunda intenção, que consiste em deixar a ferida granular de forma espontânea; esta técnica pode ser ajudada com curativos hidrocoloides para favorecer a cicatrização.

Descreve-se o caso de uma paciente idosa com três defeitos grandes faciais, na qual se optou por deixar cicatrizar por segunda intenção ajudada com curativos hidrocoloides.

\section{Bibliografía}

1. Benedetto PX, Poblete-López C. Mohs micrographic surgery technique. Dermatol Clin 2011; 29(2):141-51.

2. Magliano J, Navarrete J, Martinez M, Bazzano C. Cirugía micrográfica de Mohs en Uruguay: primeros 130 casos en carcinomas cutáneos. Rev Méd Urug 2018; 34(1):29-38.

3. Blechman AB, Patterson JW, Russell MA. Application of Mohs micrographic surgery appropriate-use criteria to skin cancers at a university health system. J Am Acad Dermatol 2014; 71(1):29-35.

4. Bichakjian CK, Olencki T, Aasi SZ, Alam M, Andersen JS, Berg D, et al. Basal cell skin cancer, version 1.2016, NCCN Clinical Practice Guidelines in Oncology. J Natl Compr Canc Netw 2016; 14(5):574-97.

5. National Comprehensive Cancer Network. Guidelines on basal cell skin cancers, version 1.2018. NCCN Guidelines 2018. Disponible en: http://www.nccn.org [Consulta: 2/2/18]

6. Garcés Gatnau JR, Catalá Gonzalo A, Alegre Fernández M, Puig Sanz L. Cierre por segunda intención de defectos cutáneos posquirúrgicos. Piel 2012; 27(7):408-15.

7. Basset-Séguin N, Chaussade V, Vilmer C. Carcinomes basocellulaires. Dermatologie 2011; 98-620-A-10.

8. Barrios E, Garau M, Alonso R, Musetti C. IV Atlas de incidencia del cáncer en el Uruguay 2007-2011. Montevideo: Comisión Honoraria de Lucha Contra el Cáncer; 2014: 82-3.

9. Barrios E, Musetti C, Alonso R, Garau M. V Atlas de mortalidad por cáncer en el Uruguay 2009-2013. Montevideo: Comisión Honoraria de Lucha Contra el Cáncer, 2015.

10. Ríos-Buceta L, Picoto A. Cirugía de Mohs. Actas Dermosifilogr 2003; 94(8):503-23.

11. Gencoglan G, Ozdemir F. Nonmelanoma skin cancer of the head and neck: clinical evaluation and histopathology. Facial Plast Surg Clin North Am 2012; 20(4):423-35.

12. Sheehan J, Kingsley M, Rohrer TE. Cirugía escisional y reparación, colgajos e injertos. En: Fitzpatrick. Dermatología en Medicina General. Tomo II, 8a ed. Buenos Aires: Médica Panamericana, 2014:2926-27.

13. López-Estebaranz JL. Tratamiento del carcinoma basocelular invasivo o la vía del erizo. Piel 2012; 27(8):425-8. 
14. Jain S, Song R, Xie J. Sonidegib: mechanism of action, pharmacology, and clinical utility for advanced basal cell carcinomas. Onco Targets Ther 2017; 10:1645-53.

15. Cebrián Carretero J, Chamorro Pons M. Principios de utilización de colgajos. Revisión de cicatrices. En: Burgueño García M, Gómez García E, eds. Atlas de colgajos locales en la reconstrucción de cabeza y cuello. España: Elsevier, 2007:89-105.

16. Burgeño García M, Arias Galli J, Gonzalez Martín Moro J. Reconstrucción de la región nasal. En: Burgueño García M, Gómez García E, eds. Atlas de colgajos locales en la reconstrucción de cabeza y cuello. España: Elsevier; 2007:157-205.

17. Fernández Suárez R, Valdivieso M, Martínez D, Mauleón C, Lázaro Ochaita P. Colgajos cutáneos-los más usados. Cir Dermatol 2001; 4(5):319-25.
18. Snow SN, Stiff MA, Bullen R, Mohs, FE, Chai WH. Second-intention healing of exposed facial-scalp bone after Mohs surgery for skin cancer: review of ninety-one cases. J Am Acad Dermatol 1994; 31(3 Pt 1):450-4.

19. Navarrete J, Magliano J, Martínez M, Bazzano C. Reconstructive methods in Mohs micrographic surgery in Uruguay: a bidirectional descriptive cohort analysis. Actas Dermosifiliogr 2018; 109(3):254-61. doi:10.1016/j.ad.2017.10.007

20. Bazzano C, Álvarez M, Martínez M. Apósitos hidrocoloidales en la cicatrización por segunda intención de heridas quirúrgicas. Rev Méd Urug 2008; 24:32-6.

21. Hien NT, Prawer SE, Katz HI. Facilitated wound healing using transparent film dressing following Mohs micrographic surgery. Arch Dermatol 1988; 124(6):903-6. 